Gynäk. Rdsch. 1978;18:153-154

\title{
Neue Lungenfunktionen und Schwangerschaft
}

G.R.

Baldwin

Zur Testung der beiden neueren Lungenfunktionsdaten CV (closing volume, pathologisch bei Stenose terminaler Bronchien in abhängigen Lungenpartien) und FVL (flow-volume loop, pathologisch bei allgemeiner Bronchialobstruktion) wurden im St. Elizabeth's Hospital in Boston, USA, 19 Gesunde zwischen 16 und 31 Jahren im 3. Schwangerschaftsdrittel und innerhalb von 6 Wochen nach der Entbindung untersucht.

Signifikante Unterschiede der Befunde während und nach Schwangerschaft waren nicht festzustellen.

Zusätzlich wurde eine Reihe konventioneller Lungenfunktionsteste ebenfalls untersucht. Dabei ergaben sich in Übereinstimmung mit der Literatur keine Unterschiede für VC (Vitalkapazität), FEV, (forciertes exspiratorisches Volu-men der 1. Sekunde), MMF (maximaler mittelexspiratorischer Flow), RV (Residualvolumen) und TLC (totale Lungenkapazität). Festgestellt wurden dage-gen signifikante Erniedrigungen von ERV (exspiratorisches Reservevolumen) und FRC (funktionelle Residualkapazität) im 3. Schwangerschaftsdrittel. Diskutiert werden Literaturmitteilungen über Messungen von R (ResistanceAtemwegswiderstand) mit verschiedenen Methoden einschliesslich der Ganzkörperplethysmographie und von C (Compliance-Lungendehnbarkeitsquotient) mit der Ösophagusdruckmethode. (Die Compliance verhielt sich dabei unter der Gravidität so wie bei Normalpatienten in liegender Körperposition.)

Kommentar

Relevante Atmungsuntersuchungen bei gesunden und atmungskranken Graviden liegen sicher noch zu wenig vor. Die hier festgestellten Verminderungen von ERV und FRC sind durch den erhöhten Zwerchfellstand erklärt. Wichtiger

1 Originaltitel: New Lung Function and Pregnancy. 